\section{Public Health Genomics}

Public Health Genomics 2016;19:352-363

DOI: $10.1159 / 000449152$
Received: July 18, 2016

Accepted: August 16, 2016

Published online: September 28, 2016

\title{
Test Pricing and Reimbursement in Genomic Medicine: Towards a General Strategy
}

\author{
Athanassios Vozikis $^{\mathrm{a}}$ David N. Cooper ${ }^{\mathrm{b}}$ Christina Mitropoulou ${ }^{\mathrm{c} d}$ Manousos E. Kambouris ${ }^{\mathrm{c}}$ \\ Angela Brand $^{\mathrm{e}}$ Vita Dolzan $^{f}$ Paolo Fortina ${ }^{g}$ Federico Innocenti ${ }^{\text {h }}$ Ming Ta Michael Lee ${ }^{\mathrm{i}}$ \\ Lada Leyens $^{\mathrm{e}}$ Milan Macek Jr ${ }^{\mathrm{j}}$ Fahd Al-Mulla $^{\mathrm{k}}$ Barbara Prainsackl Alessio Squassina ${ }^{\mathrm{m}}$ \\ Domenica Taruscio $^{n}$ Ron H. van Schaik ${ }^{d}$ Effy Vayena ${ }^{\circ}$ Marc S. Williams ${ }^{i}$ George P. Patrinos ${ }^{p}$ \\ a Laboratory of Health Economics and Management, Department of Economics, University of Piraeus, Piraeus, \\ Greece; ${ }^{b}$ Institute of Medical Genetics, School of Medicine, Cardiff University, Cardiff, and ${ }^{\mathrm{C} T h e}$ Golden Helix \\ Foundation, London, UK; ' d Department of Clinical Chemistry, Erasmus University Medical Center, Rotterdam, and \\ e Maastricht Economic and Social Research Institute on Innovation and Technology (MERIT), Graduate School \\ of Governance, Faculty of Humanities and Sciences, Maastricht University, Maastricht, The Netherlands; ${ }^{f}$ School \\ of Medicine, University of Ljubljana, Ljubljana, Slovenia; ${ }^{9}$ Kimmel Cancer Center, Thomas Jefferson University,

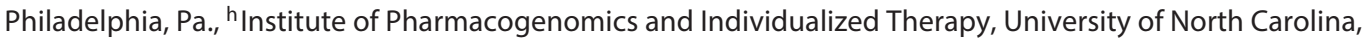 \\ Chapel Hill, N.C., and 'Genomic Medicine Institute, Geisinger Health System, Danville, Pa., USA; 'ं Institute of Biology \\ and Medical Genetics, Charles University Prague 2nd Faculty of Medicine and University Hospital Motol, Prague, \\ Czech Republic; ' Molecular Pathology Unit, Health Sciences Center, Faculty of Medicine, University of Kuwait, Safat, \\ Kuwait; 'Department of Global Health \& Social Medicine London, King's College London, London, UK; \\ mDepartment of Biomedical Sciences, School of Medicine, University of Cagliari, Cagliari, and national Centre for \\ Rare Diseases, Istituto Superiore di Sanità, Rome, Italy; ${ }^{\circ} \mathrm{Health}$ Ethics and Policy Lab, Epidemiology Biostatistics

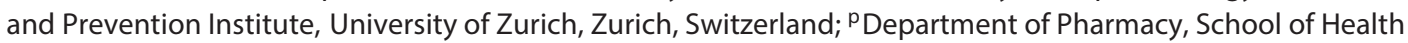 \\ Sciences, University of Patras, Patras, Greece
}

\section{Key Words}

Genomic medicine $\cdot$ Pharmacogenomics $\cdot$ Public health genomics · Health economics · Whole genome sequencing . Genomics education · Policy - Reimbursement

\begin{abstract}
This paper aims to provide an overview of the rationale and basic principles guiding the governance of genomic testing services, to clarify their objectives, and allocate and define responsibilities among stakeholders in a health-care system, with a special focus on the EU countries. Particular attention is paid to issues pertaining to pricing and reimbursement
\end{abstract}

\section{KARGER}

(c) 2016 S. Karger AG, Basel

E-Mail karger@karger.com

www.karger.com/phg policies, the availability of essential genomic tests which differs between various countries owing to differences in disease prevalence and public health relevance, the prescribing and use of genomic testing services according to existing or new guidelines, budgetary and fiscal control, the balance between price and access to innovative testing, monitoring and evaluation for cost-effectiveness and safety, and the development of research capacity. We conclude that addressing the specific items put forward in this article will help to

\section{Athanassios Vozikis}

Laboratory of Health Economics and Management

Department of Economics, University of Piraeus

80 Karaoli \& Dimitriou Street, GR-18534 Piraeus (Greece)

E-Mail avozik@unipi.gr

George P. Patrinos

Department of Pharmacy, School of Health Sciences

University of Patras, University Campus, Rion

GR-26504 Patras (Greece)

E-Mail gpatrinos@ upatras.gr 
create a robust policy in relation to pricing and reimbursement in genomic medicine. This will contribute to an effective and sustainable health-care system and will prove beneficial to the economy at large.

(c) 2016 S. Karger AG, Basel

\section{Introduction}

At a time of unprecedented technological advancement, genomic medicine (an important part of personalized and precision medicine strategies) holds out the promise to detect the onset of disease, ideally at its earliest stages, either presymptomatically or through the determination of individual risk, in an effort to forestall (prevent) the progression of disease and to individualize drug treatment modalities. At present, this can be performed in a handful of cancers, in monogenic disorders such as the hemoglobinopathies and cystic fibrosis etc., and for over 100 drug treatment modalities [1]. Genomic medicine aims to improve the shared medical decision-making process and to rationalize drug prescription for the benefit of both the patient and the national healthcare system, by taking advantage of an individual's unique genetic/genomic profile. Genomic medicine has the potential to (a) shift the emphasis in medicine from clinical/therapeutic intervention to prevention, (b) direct and/or inform the selection of optimal therapies and reduce trial-and-error prescribing, (c) help to avoid adverse drug reactions, (d) improve quality of life, (e) reveal additional or alternative uses for medicines and drug candidates and help in the selection/design of novel therapeutics or therapeutic regimes (re-tasking), and (f) aid in containing the overall cost of health care in the medium to long term [2].

Genomic medicine also promises to improve the quality of clinical care, while ideally lowering health-care costs. Although scientific challenges still remain and both the cost and time required to perform genome sequencing continues to drop, the economic issues and policy making still present a serious hurdle [3]. The decision to introduce genomic medicine into the public health-care system is dependent upon a range of factors and will require the combined efforts of multiple stakeholders in society (innovators, research organizations and academic centers, entrepreneurs, funders and payers, citizens, both healthy individuals and patients, regulators, policymakers, and legislators). It would appear that many key stakeholders are generally favorable to the implementation of genomic medicine [4-6]. However, all necessary policies

Test Pricing and Reimbursement in Genomic Medicine and measures should be implemented to foster the overall positive attitude of most stakeholders towards genomic medicine, while at the same time ensuring that genomic medicine is implemented in an efficient and effective manner that upholds the spirit of solidarity in health-care systems in most countries. If this is done, then the remaining stakeholders, who might hold a neutral-to-negative opinion, will also be content and supportive [3].

The most important factors required for the successful implementation of genomic medicine into the public health-care system are: (a) affordability, (b) acceptability, fairness, solidarity and appropriateness, (c) appropriate knowledge and education (for clinicians and patients) available, (d) political engagement and willingness to change existing health care, (e) appropriate policy and legislation, ( $\mathrm{f}$ ) demonstration of proven and undisputed clinical efficacy, and (g) of demonstrated cost-effectiveness [7-9]. If payers are unwilling to reimburse the costs of genomic testing services, progress towards incorporation and implementation will be stalled. Reimbursement decisions in relation to genomic testing are complicated, and although genomic testing has been performed for more than 20 years, the respective decision-making process is still evolving $[10,11]$.

In the United States, the Centers for Medicare and Medicaid Services (CMS) manage the Clinical Laboratory Improvement Amendments (CLIA) program, which inspects and regulates clinical laboratories, including those performing genomic testing [12-14]. Several US government agencies are currently working towards the development of regulatory standards for genomic testing laboratories and the comprehensive integration of genomic testing into routine medical practice. The Centers for Disease Control and Prevention has an advisory group specifically focused on the CLIA regulations (Centers for Disease Control: Genetic Testing Policy), as well as projects studying the validation of genomic tests and the integration of these tests into clinical practice (Centers for Disease Control - Genetic Testing). In addition, the Secretary's Advisory Committee on Genetics, Health and Society (SACGHS) issued a comprehensive report summarizing the issues surrounding the reimbursement of genomic tests (see: Coverage and Reimbursement of Genetic Tests and Services) $[9,15]$. The National Human Genome Research Institute (NHGRI) hopes to serve as a resource for advancing genomic medicine by assisting all types of payers in their efforts to evaluate emerging genomic tests for reimbursement, and by promoting research into the health benefits and cost-effectiveness of genomic testing [16]. 
In the EU, the regulatory structure for the reimbursement of medical devices differs from country to country (recent regulations brought in in vitro diagnostics that require stricter clinical evidence). In Germany, it is administered by Der Gemeinsame Bundesausschuss (GBA), in France by La Haute Autorité de Santé (HAS), in the UK by the National Health Service (NHS), in Italy by Il Servizio Sanitario Nazionale (SSN) and in Spain by El Instituto Nacional de la Salud (INS). Consequently, the health-care procedure reimbursement structure for European nations also differs from country to country due to differences in health-care budgets, health-care policies, etc. [17]. However, all EU countries follow the EU directive on medical device regulations. Within this directive, a medical device must have the Conformité Européenne (CE) mark to be sold within the respective member countries denoting some sort of EU harmonization [12-14]. However, this is not the only prerequisite for a device to be sold in the EU since many member states have also adopted complex reimbursement policies (requiring the device to be included in an approved reimbursement list of diagnostic amenities).

\section{Coverage, Pricing and Reimbursement Strategies for Genomic Tests}

The reimbursement structures in the United States and European countries are influenced by spiraling health-care costs, increasing patient waiting times, and deficit financing for their burgeoning health-care expenditure. This has led to restrictions in the amounts public payers or managed care organizations are reimbursing, with respect to expensive new genomic tests and molecular diagnostic procedures.

While cost-effectiveness may or may not be considered by payers in their decision-making process relating to these high-value tests, the impact of direct cost savings is important for some payers, particularly in relation to the use of these tests in the context of the avoidance of inappropriate or unsafe drug therapy [12-14, 17]. However, the EU's reimbursement environment is not uniform since each member state has its own policies, with reimbursement being approved by either private or public insurance companies or a combination of the two. Approval for reimbursement from public health providers often requires lengthy negotiations. The potential differences across member states are compounded by the problem of centralized versus decentralized national systems, including the tendency of most EU nations to review/authorize tests at the local level. This local approach to coverage and reimbursement assessment of genomic testing, compared with national level reviews for most drugs, can present substantial barriers to consistent market access for genomic/pharmacogenomic tests and services [12-14].

Product pricing also displays marked differences due to various factors such as supply-demand fluctuations, differences in various government tax rates (for example VAT, customs) etc. Thus, France has reduced the rate of VAT payable on medical devices, whereas other countries including Germany have maintained the maximum rate of VAT. Moreover, there are differences in health technology assessment (HTA) systems both between and within countries that would benefit from standardization; at present, there is no precedent for value capture, as the future regulatory framework for high-risk tests, including those used in genomic medicine, is likely to evolve $[18,19]$.

\section{Components of the Proposed Strategy for Pricing and Reimbursement}

The ideal strategy for pricing and reimbursement in genomic medicine should incorporate the following objectives (fig. 1):

1 Universal access to essential genomic testing for all, and at acceptable prices for the health-care system

2 Sufficient regulation to ensure safety, efficacy, quality, fairness and solidarity, while allowing space for innovation necessary to move the field forward

3 Appropriate use of genomic tests and information by physicians, according to patients' needs and clinical utility/action ability of testing outcomes

4 Investment in both human resources and capital for the research into the field of genomic medicine, evaluation of novel and existing diagnostic procedures, and monitoring of patient safety

These objectives are discussed in detail below from the perspective of the European Union, although most of the principles presented will have applicability to decision makers in other systems.

Universal Access to Essential Genomic Testing for All, at Acceptable Prices for the Health System

By definition, essential genomic tests are those tests that satisfy the priority health-care needs of a given population. Essential genomic tests, like essential medicines, are selected with due regard to disease prevalence and public health relevance, evidence of clinical efficacy and safety, and comparative costs and cost-effectiveness. 
Fig. 1. The framework of the sequence of stages a new genomic (companion) diagnostic test has to pass before a citizen-patient may obtain access to it.

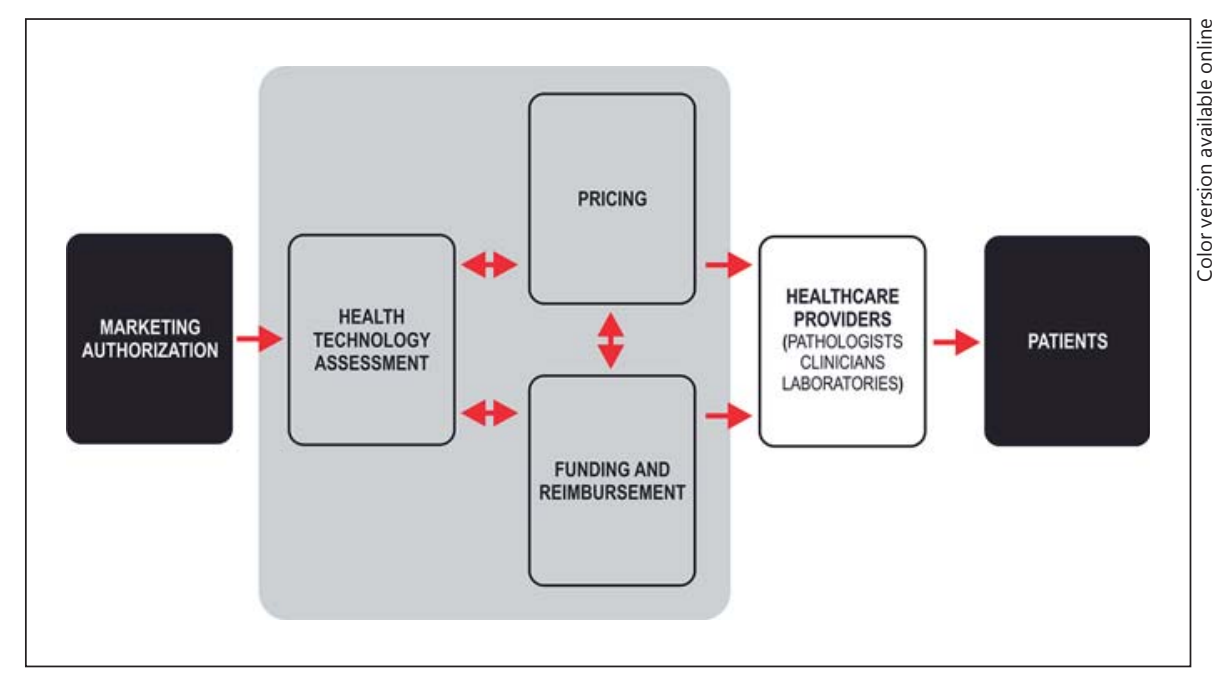

Availability

Ideally, the availability of genetic testing should be the right of all residents (insured or not/not tied to citizenship, as applied in the EU countries) to ensure the safe use of medication and should be: (a) made available in a timely manner, (b) distributed according to therapeutic need, and (c) accessible irrespective of locality, age, gender or income. Fulfillment of these prerogatives (along with supporting clinicians and patients to promote good decision making) will lead to greater health and patient safety, as well as greater efficiency and sustainability of genomic medicine and of health systems. Currently, genomic tests may be prescribed on the basis of the patients' ability to pay, rather than need, therapeutic rationale or cost-effectiveness [20]. This policy proposal addresses the problems of high out-of-pocket payments and expenditure, and deviation from international standards of care and equitable access.

In order to avoid high out-of-pocket payments, it is first necessary to select the essential genomic tests that would be made available and reimbursed under the positive medical devices' list. Driven, kept relevant and regularly updated by clinical guidelines, this process would contribute to a more rational prescribing regime (as was the case with drug prescription), less confusion and greater familiarity with genomic testing amongst diagnostic laboratories and providers. On the whole, the development of a positive medical devices' list should lead to better health outcomes with cost containment in both the medium and long term [21]. As will be described later, the classification of every genomic test should be documented by cost-effectiveness analyses with provisions to use HTAs in the decision-making process.

Test Pricing and Reimbursement in Genomic Medicine
The key stakeholders tasked with ensuring the availability and affordability of genomic tests are the Ministries of Health, regulatory bodies, payer organizations, medical device manufacturers, wholesalers, academic and other research institutes, pharmaceutical companies, pharmacies, hospitals and clinics, public and private diagnostic laboratories and physicians [22]. The European Parliament Directives (in the next few years, they will be changed to regulations so as to avoid different national interpretations) set the rules on marketing authorizations, as well as on advertising, product quality and safety, and labeling regulations. These policies complement those already made at the national level, and especially those outlined in our policy proposal, with regard to pricing, access, research, and monitoring.

\section{Affordability}

Affordable prices are crucial to ensuring that the population as a whole has access to essential genomic tests. Increasing affordability should be a key commitment of government to guarantee that pecuniary issues do not constitute barriers to people's access to genomic testing. The government and regulatory bodies should address the affordability of essential genomic tests, such as pharmacogenomic tests, as a policy concern in the context of both pricing and reimbursement.

Only an effective, transparent, sustainable and robust pricing system can guarantee affordable prices and hence access to genomic tests for the entire population. Under such an arrangement, the pricing of genomic tests is accomplished through merit and supply and demand, which ensures that pricing decisions take into account the 
comparative effectiveness of new genomic tests and their incremental effects relative to their incremental costs, as components of a cost-effectiveness analysis. The pricing process should be transparent, and all stakeholders should have access to all the relevant information that was used to determine prices, most likely through negotiation under a comprehensive HTA process. Each country's Medical Devices regulatory authority or HTA Agency needs to build capacity in terms of infrastructure, skills and expertise to be able to fulfill the task of determining the appropriate pricing of genomic tests and to take a strong stand in disputes with manufacturers or representatives over the determination of prices, always recognizing the complications and bottlenecks of the existing system.

The pricing system should ensure that, while competitive market rules are not violated, public and private payers and insurers are protected from excessive expenditure that could act as a barrier to patient access, such as high out-of-pocket payments. Pricing policies should aim to reduce monopoly power granted to manufacturers in order to contain costs in the diagnostic market. The pricing system should support a sustainable environment for such products that achieves an optimal mix between health and commercial policy. Cost containment measures should reflect these priorities and the development of a sound policy should take precedence over price regulatory measures to achieve the goal of long-term sustainability of the health-care system.

An effective, transparent and sustainable reimbursement system for all clinical products guarantees reasonable cost sharing for the population, while ensuring their access to these tests and services. Adequate levels of copayments (which are paid in part by the patients/citizens themselves) and co-insurance ensure that cost and expenditure reduction goals are met in every country while not compromising patient access in terms of affordability. The criteria of genomic test classification for the positive list, including essential genomic tests by default, should be carefully controlled and regularly reviewed, especially in relation to the emergence of clinical, economic, fiscal or other criteria, and how these different criteria should be weighed against each other in the decisionmaking process. Price bulletins should be issued in order to keep stakeholders informed of price fluctuations. In addition, a negative list (like those already in place in several countries) of genomic tests should also be developed to include those genomic tests that fail to meet certain scientific standards, such as is currently the case with nutrigenomic testing, as well as tests that aim to determine athletic performance, intelligence criminality, personali- ty, genomic identity testing, etc. [23-25]. For such tests, no co-payments should be allowed, whether through public or private insurance schemes; out-of-pocket payments should be the only means to purchase them.

It is also of the utmost importance that government remains committed to the public financing of health services. At present, a course of privatization and market shift is becoming increasingly apparent even in those European countries traditionally oriented toward public health care provision (e.g. France, UK, Czech Republic and latterly Greece). Given that in most cases, public health resources are limited, it is necessary to carefully (re)define government policy and select priorities for public funding on that basis. The establishment of an HTA agency could also contribute to the selection of priority areas for the allocation of limited public health funds.

A first action would be to set up a pricing committee within a country's HTA Agency, that would oversee the pricing of all genomic tests which are in strict adherence to EU (or other area) legislation (though some argue that pricing needs to be based on competition and market rather than committees). Although organized within the country's medical devices regulatory authority or HTA agency, the pricing committee should have a sufficient number of qualified members of staff to conduct background checks and maintain databases in order to advise appropriate prices for all new (or existing) genomic tests entering the market, aiming to allow reimbursement by public insurance funds. The committee should fulfill its role in a timely fashion and in a robust way. To achieve these goals, it will be necessary to define the committee's terms of reference, member composition, including assessment of necessary content expertise, governance, lines of accountability, and support by a secretariat, which would include in-house capacity for data searching, database maintenance, and cooperation with the stakeholders. The pricing committee, just as in the case of medicines, should also participate in pharmaceutical and medical devices policy cooperation EU committees (or forums) in order to ensure that the committee has access to information about ongoing and planned activities in the field of pricing policy at community level while adhering to the transparency directive.

Another key step would be to merge all currently existing committees that are involved in the reimbursement process under the umbrella of a positive list and reimbursement committee. This committee would oversee genomic test reimbursement in order to reform the usually highly fragmented system that is currently in place in 
many countries. The reimbursement committee would need to act according to a governance structure with rules pertaining to responsibility and accountability that would have to be clearly defined.

With respect to specific genomic tests, hospitals and the Public Insurance Funds/Payers might employ a tendering system to promote price competition between manufacturers or, most importantly, distributors. However, this method of procurement has sometimes been associated with delays in patient access, as companies that have lost out in tendering have reacted by contesting the award of a contract. While tendering remains a viable system for pricing and procurement of genomic tests, the current system must be streamlined and made more transparent. To be sustainable, this process must be repeated fairly regularly, ideally on an annual (or biennial) basis, depending on the innovation stepping.

Proposed Actions for Objective 1

1 Expand the range of evidence-based clinical guidelines (prescribing recommendations) taking into account international standards. These must be an integral part of the e-prescribing module of a national health information system.

Relevant stakeholders: National medicine agency, the medical devices regulatory authority or HTA agency, positive list committee, public insurance funds/payers physicians, international partners.

2 Set up a pricing advisory committee within the HTA Agency that would oversee the pricing of all new or existing genomic tests with strict adherence to EU (or other area) legislation.

Relevant stakeholders: The medical devices regulatory authority or HTA agency, public insurance funds/payers, ministry of health, patient representatives.

3 Merge all currently existing reimbursement committees under the umbrella of a positive list and reimbursement committee.

Relevant stakeholders: The medical devices regulatory authority or HTA agency, ministry of health.

4 Streamline and make transparent the procurement and pricing processes in order to avoid launch delays of innovative tests.

Relevant stakeholders: The medical devices regulatory authority or HTA agency, public insurance funds/payers, medical devices industry, wholesalers and private pharmacies, genetic laboratories.

5 Revise the current positive list to align with treatment protocols in order to rationalize procurement of innovative genomic tests.

Test Pricing and Reimbursement in Genomic Medicine
Relevant stakeholders: The medical devices regulatory authority or HTA agency, public insurance funds/payers, medical devices industry.

6 Develop, implement and enforce expeditious and transparent tendering and review processes for specific genomic tests.

Relevant stakeholders: The medical devices regulatory authority or HTA agency, public insurance funds/payers, medical devices industry.

7 Maintain government commitment to public financing of health services.

Relevant stakeholders: Ministry of health, public insurance funds/payers.

Sufficient Regulation to Ensure Safety, Efficacy, Quality, Fairness, and Solidarity, while Allowing Space for Innovation Necessary to Move the Field Forward

Robust regulation is a key parameter to ensure test safety, efficacy and quality and should be a product of strong cooperation between all relevant stakeholders. Quality assurance is the responsibility of all the stakeholders involved in the medical device supply chain, albeit in different ways. Manufacturers are responsible for developing and manufacturing good quality products and adhering to good manufacturing practice. The medical devices regulatory authority must ensure that tests approved for prescribing (and reimbursement) are first evaluated and registered appropriately, that manufacturers comply through licensing and inspection and that the test quality is ensured and maintained in the supply system. This includes the assessment of good storage and distribution practices in addition to continual monitoring of the quality of testing devices and procedures in the distribution chain. Procurement agencies should ensure that genomic tests are selected carefully, purchased from reliable sources, namely distributors and/or wholesalers, inspected at the time of delivery, and stored and transported appropriately.

Safety assurance is the second key consideration for regulatory bodies. Safety is reviewed during the market approval and registration process, but postmarketing monitoring has to be in place to reliably record and assess adverse reactions. In the EU, it is the EU Medical Device Vigilance System that collects and manages data on the safety of medicines and uses them to make decisions with regard to safety issues [26]. A similar process must also be put in place for genomic testing services, to address issues pertaining to accuracy and/or testing reliability. 
While regulation for quality and safety is important, it must also be recognized that in a rapidly changing field such as genomics, traditional approaches to regulation may not be able to respond quickly enough to the changing evidence. This has the potential to impede innovation [27]. Therefore, careful attention must be paid to balancing regulations to assure quality and safety while preserving the opportunity for innovation.

Regulatory decisions therefore result from substantial cooperation and consultation among all the relevant stakeholders in the medical device market, particularly in relation to the timely implementation and monitoring of the relevant provisions. Manufacturers are responsible for providing high-quality products, whereas physicians play a key role in following medical guidelines that ensure appropriate prescribing. Government institutions and regulators are responsible for the promotion of cooperation among all the stakeholders and the involvement of these parties in the decision-making process.

\section{Actions for Objective 2}

1 Quality assurance has to be the responsibility of all the stakeholders in the supply chain.

Relevant stakeholders: Medical devices industry, ministry of health, public insurance funds/payers, quality assurance organizations, physicians, pharmacists, wholesalers, and all the regulatory bodies.

2 Adherence to the EU Medical Device Vigilance System needs to be enforced.

Relevant stakeholders: The medical devices regulatory authority, public insurance funds/payers, ministry of health and all the regulatory bodies.

3 Regulatory decisions need to build on substantial cooperation between all the stakeholders.

Relevant stakeholders: The medical devices regulatory authority, ministry of health, public insurance funds/payers, all the regulatory bodies, physicians, the medical devices industry, wholesalers, and pharmacists.

Appropriate Use of Genomic Tests and Information by Physicians, according to Patients' Needs and Clinical Utility/Actionability of Genomic Testing Outcomes

Physicians have the responsibility for prescribing genomic tests rationally, ensuring appropriate, adequate and cost-effective care, while informed and empowered patients should play an equally important role based on their personal circumstances and preferences. Prescribing genomic tests rationally requires the provision of best practice recommendations and relevant information to physicians. A continued expansion, enhancement and leveraging of the e-prescribing systems (see next paragraph) is essential for more effective, efficient, and appropriate prescribing of tests to all citizens, e.g. as a screening policy for high-risk citizens. This system will monitor physicians' prescribing, providers' implementation and pharmacists' distribution records in order to rationalize the provision of genomic tests [28]. A good example is the use of disease-specific 'Clinical Utility Gene Cards' which establish a priori peer-reviewed criteria for indication of genomic testing [29]. Another issue which needs to be dealt with is the uncontrolled indication of cross-border genomic testing [30].

The health-care industry is witnessing an increased adoption of e-prescribing systems due to the pressing need to curtail health-care costs, while maintaining the quality of care provided to patients and reduce medication errors and adverse drug events. The market is also witnessing a growing demand for integrated e-prescription systems, facilitating the health-care community to shift towards the implementation of a complete electronic health record system [31].

Important measures for rational use from the side of suppliers are the prescribing of genomic tests on the basis of international classification (coding) and their provision to citizens-patients according to existing guidelines. The former depends critically upon physicians' access to standardized prescribing protocols across therapeutic categories. Physicians and patients must work together to understand the treatment and the diagnostic options available. Therefore, evidence-based medicine must be provided to patients at the level of the physician and the pharmacist.

\section{Actions for Objective 3}

1 Expand and continuously update the e-prescribing system; integrate this IT database with patient record databases currently existing (or in development).

Relevant stakeholders: The medical devices regulatory authority, public insurance funds/payers, ministry of health and all the regulatory bodies, physicians, pharmacists, and international partners.

2 Prescriptions made according to international prescribing protocols, with a view to engaging in international coding-based prescribing.

Relevant stakeholders: The medical devices regulatory authority, public insurance funds/payers, physicians, and international partners.

3 Educate clinicians with respect to the favorable use of evidence-based (in terms of safety and efficacy) medi- 
cine to meeting patient's needs; foster collaborative doctor-patient relationships in order to make prescribing more efficient.

Relevant stakeholders: Physicians, pharmacists, patients.

Investment in Both Human Resources and Capital

for Research into the Field of Genomic Medicine,

Evaluation of Novel and Existing Diagnostic

Procedures, and Monitoring of Patient Safety

Investments in human capital and infrastructure are key components to promote research in the field of genomic medicine, evaluate novel and existing diagnostic procedures, and monitor patient safety to promote effective patient care, affordability of the health-care system and profitability for the industry. Research is a critical component for health-care systems in order to allocate resources appropriately and evaluate performance optimally. It is especially necessary for the genomic medicine sector, which has the added requirement of sufficient $\mathrm{R} \& \mathrm{D}$ investment for newer, innovative approaches and products. Thus, research not only has the potential to make savings and recoup expenditure, thereby making efficiency gains for the health sector, but also to contribute to the economy as a whole through the development of patentable technologies.

Investment in $\mathrm{R} \& \mathrm{D}$ is partly a function of the healthcare system, of the national priorities for health-care research and of a whole host of factors, both financial and nonfinancial, that define national policy. Significant research investments are made by manufacturers, and this may be encouraged through both industrial and health policy. A national policy platform may help to encourage investment in $\mathrm{R} \& \mathrm{D}$, for example by promoting a more flexible and responsive regulatory framework to attract clinical trials.

Rational use of resources relies upon the availability of clinical cost-effectiveness or cost-benefit or relative effectiveness data. HTA activities can contribute significantly in this direction, for example by (a) contributing to the making and updating of positive, negative and high-cost lists of genomic tests by the payers, (b) helping to divert public health funding from less cost-effective services towards diagnostic tests and pharmacotherapies with greater diagnostic, prognostic and therapeutic values, respectively, (c) specifying clinical and prescribing protocols based on clinical cost-effectiveness criteria, and (d) advancing e-health systems.

Test Pricing and Reimbursement in Genomic Medicine
Actions for Objective 4

1 Identify and address gaps in the regulatory framework that will help to attract R\&D investment.

Relevant stakeholders: The medical devices regulatory authority, public insurance funds/payers, ministry of health, pharmaceutical companies, medical associations, other government departments.

2 Examine the scope for the identification of priorities for health research and the creation of a national platform for the same.

Relevant stakeholders: The medical devices regulatory authority, public insurance funds/payers, ministry of health, academic institutions, other government departments.

3 Actively pursue the use of HTA to conduct cost-effectiveness or cost-benefit or relative effectiveness analyses for more informed decision-making on genomic testing cost reimbursement.

Relevant stakeholders: The medical devices regulatory authority, public insurance funds/payers, domestic and international academics/experts.

4 Physicians should utilize their clinical expertise to assist/contribute in the creation and adoption of clinical guidelines and corresponding prescribing protocols, to be integrated into the e-health system.

Relevant stakeholders: Public insurance funds/payers, physicians, ministry of health.

\section{Public Policy Concerns}

\section{Funding and Pricing of Highly Innovative Genomic Tests}

Although lower prices may emerge from the development of increasingly efficient and widespread technologies, it is crucial that reimbursement levels, which have been set unilaterally by policymakers in most European countries will not only ensure massive access to high quality tests but also continue to allow for the development of a pipeline of innovative tests that require substantial risk-based research (defined as the uncertainty of the investment in innovation). Many genomic tests, particularly those complex predictive tests that require prospective clinical trials, are not funded or reimbursed appropriately. Such lack of funding and reimbursement inevitably tends to inhibit access, as laboratories are incentivized to offer those tests that are better reimbursed and are most reluctant to offer tests that might be unprofitable; the unfortunate consequence of this is that some treatment pathways may not be appropriately explored 
Fig. 2. Potential funding sources and mechanisms for genomic (companion) diagnostics. $\mathrm{DRG}=$ Diagnosis-related groups.

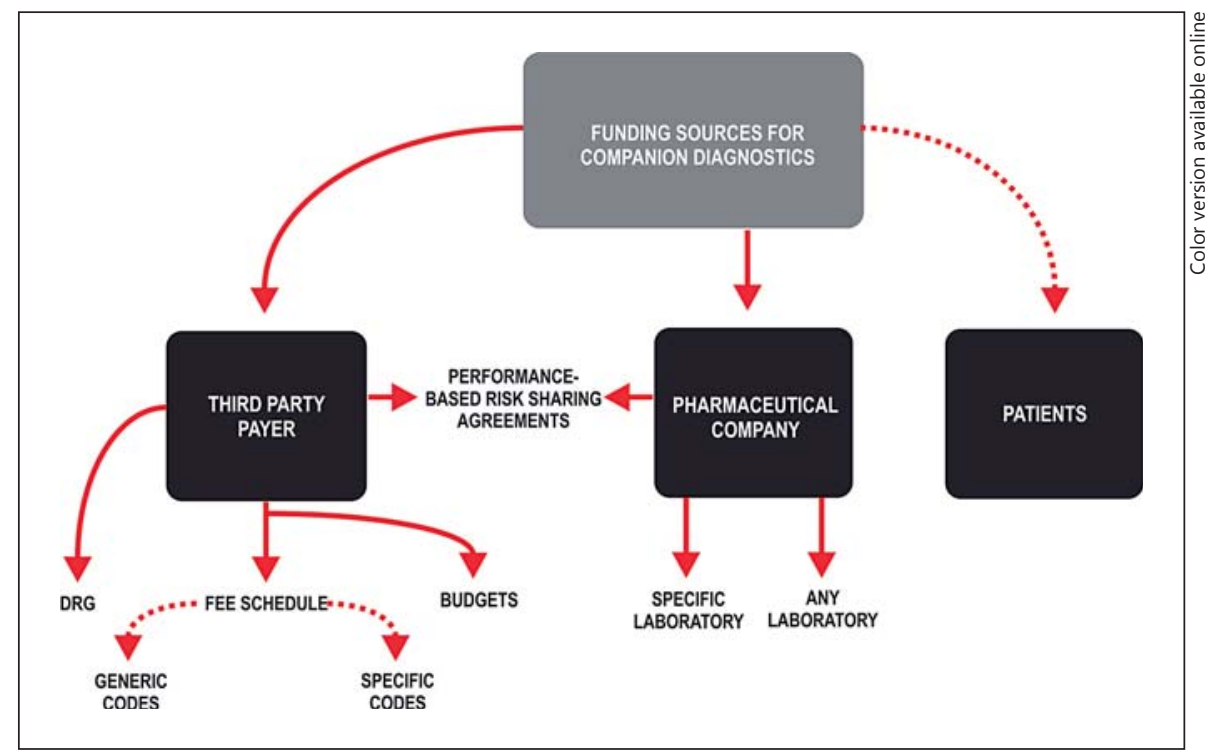

with personalized diagnostic information. Although this may present some hurdles for the private sector, regulation and EU harmonization are still required so as to minimize the impact of the 'free-market' shortcomings in the health-care sector. Moreover, the opacity of the current reimbursement environment acts as a disincentive to research and development investment in innovative diagnostics and, by extension, diagnostic-therapeutic combinations [32]. As noted above, the regulatory framework must also be sufficiently flexible to manage the rapidly changing environment while not stifling innovation [27]. Generally, funding can either be provided by the healthcare system, or by a medical device company or by the patients (fig. 2).

Recommendation: HTA processes within a country should be coordinated in a way that allows a single integrated HTA report for diagnostic companion products.

Reason: Genomic diagnostics have no direct health improvement effect but potentially generate downstream health effects when used as indicated to inform therapeutic options [26]. Making wise policy decisions in this area is the single biggest challenge for all stakeholders.

\section{Evaluating Diagnostic, Prognostic, and Predictive \\ Genomic Tests}

HTAs represent a rapidly growing area of European government policy, and one which has accelerated over the last few years as governments seek to contain costs. However, most of the paradigms for health care appraisal and technology assessment have been developed for the purpose of comparing procedures or drug therapies. Today, tests are evaluated in the context of a much broader concept of their 'clinical utility', namely cost-effectiveness, budget impact, priorities, etc. Although most people would agree that genomic tests are beneficial by having an impact both on patient management and the delivery of treatments, many are still concerned that processes underlying 'clinical utility' assessments are neither clear nor predictable. More objective and reliable standards for these evaluation processes need to become broadly accepted. Since many argue that reimbursement should be tied to value, 'value' (i.e. utility for the patient, economies for the payer) needs to be acceptably defined, taking into consideration how difficult it has been to consider all factors involved in 'value' and to implement value-based pricing [33].

Quality can be improved by employing quality assurance measures (fig. 3). The meaning of different quality endorsements is often confusing [34, 35]:

Recommendation: All laboratories performing genomic diagnostic tests should be required to be accredited, and existing external quality assessment schemes should be extended both at National and International levels.

Reason: Improving genomic testing quality reduces the number of misclassifications, such as incorrect test results. Misclassifications cannot benefit from a treatment diagnosis or who may even be at risk of harm by it being treated nonetheless. Accreditation is the most extensive quality endorsement, covering test quality inside 


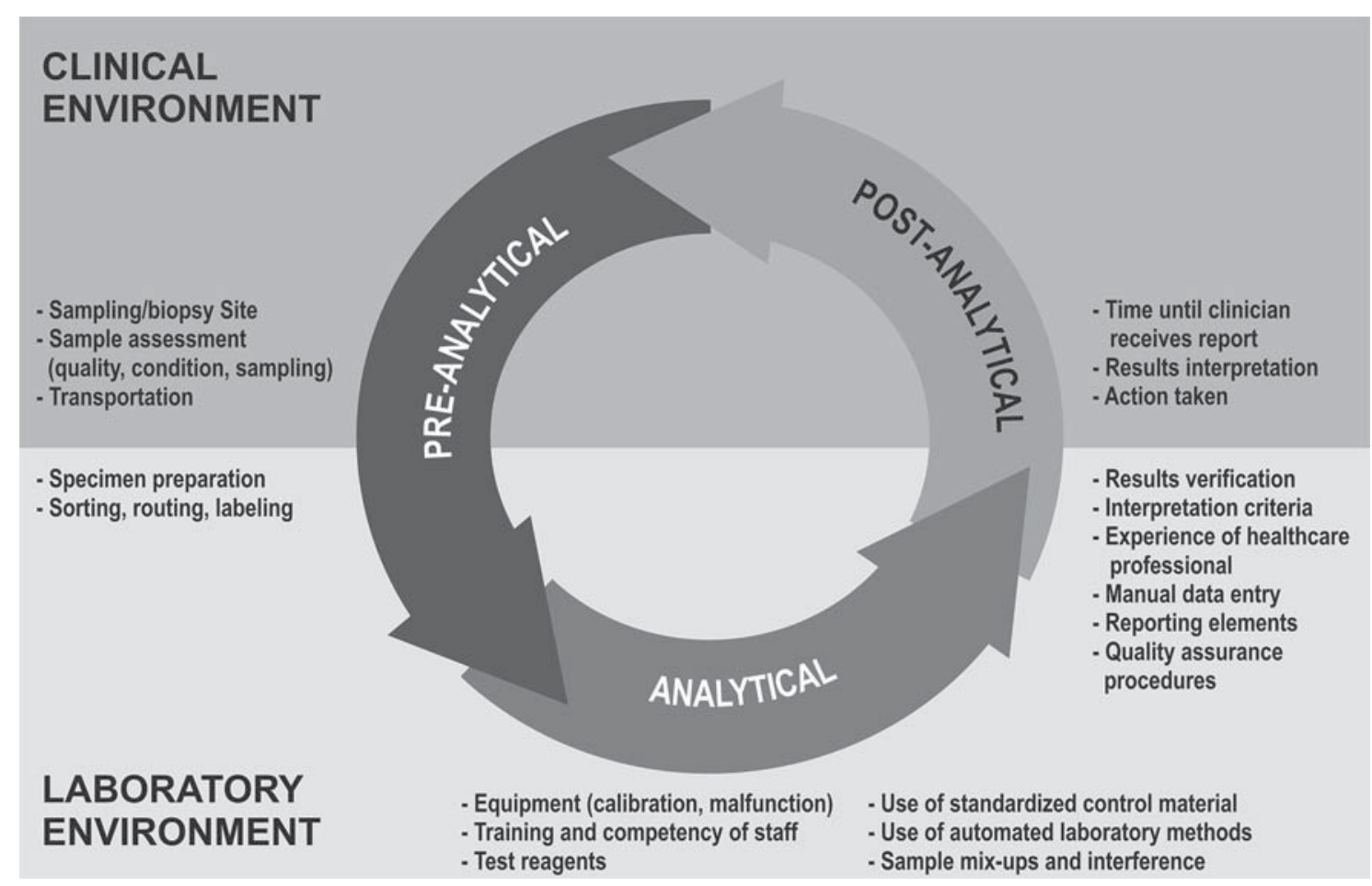

Fig. 3. Stages of the testing process and sources of variability inside and outside the genomic testing laboratory.

the laboratory and at the interface with clinicians. Accreditation standards include an obligation to participate in external quality assessment schemes. External quality assessment schemes have been shown to increase the accuracy of genomic diagnostic testing [36].

\section{Incentives for Genomic Medicine}

There will also be areas where genomic medicine could have a major impact on public health, but unfortunately traditional funding, pricing or reimbursement systems fail to provide enough incentives for test development. These areas include funding (a) the education of physicians (such as genomics education) and increasing literacy in genomics and genomics for patients and the general public [37, 38], (b) allied professionals such as genomic counselors, and (c) rewarding/creating incentives to develop new tools that could revolutionize some therapeutic areas [33]. It would be desirable to conduct a survey among clinicians in Europe to establish if there are any concerns with regard to drug-diagnostic companion products. This is further highlighted by the fact that many interviewed expert stakeholders disagreed about the role of clinicians [4]. Some stakeholders reported that clinicians are most reluctant to adopt companion diagnostics even when funding is guaranteed, whereas others reported that clinicians' adoption is not a barrier to access at all [39]. Whichever, close links between pathologists and clinicians help to increase clinicians' awareness about the kind of tests available and how these are appropriately used and interpreted. Furthermore, clinicians have an important impact on testing quality during the preanalysis stage. Finally, close links are helpful when informal advice for patients is needed.

On the other hand, patients rely traditionally on their doctors' professional opinion and generally follow their advice. In recent decades, the clinician-patient relationship has evolved into a partnership model. Indeed, in a recent survey, roughly $75 \%$ of European patients, particularly those of younger age, wished to play a more active role in health-care decision-making [40]. For these patients, it is crucial to have easy access to reliable information about their own disease/condition, and treatment options in clear and nontechnical language. This applies in particular to the complex field of genomic medicine. 
Recommendation A: Further research should investigate the knowledge and attitude of European clinicians towards genomic testing services.

Reason: Clinicians are gatekeepers for patient access to drug-diagnostic companion products. In sharp contrast to their importance for patient access, comparatively little is known about the attitudes and behaviors of European physicians.

Recommendation B: Collaboration between clinicians and pathologists in the area of genomic medicine should be increased.

Reason: A survey of US clinicians revealed that one reason for not using pharmacogenomics is a lack of knowledge as to what tests are available, how to procure them, when to use them, how to interpret the results and how to apply them in the context of an individual patient [41]. Similar results have also been forthcoming from $\mathrm{Eu}-$ ropean countries [42]. Furthermore, it has been shown that the preanalysis stage outside the laboratory is crucial for obtaining accurate test results. Increased collaboration leads to an exchange of knowledge, lowers the threshold to ask for advice and generates awareness about the available testing options.

\section{Conclusions and Future Perspectives}

Genomic testing presents special challenges to pricing and reimbursement science because of the rapid evolution of new technologies and the lack of a clear understanding by all stakeholders of the requirements for inclusion in coverage policies. Coverage for genomic tests has been a complicated issue, as no consensus exists as to what should be covered; thus, payers must consider options such as bundled payments or risk-sharing agree- ments, so that genomic tests are accompanied by treatment and an entire continuum of care. However, it is not known whether that would increase or decrease the uptake of the offered test or how it would affect implementation [43].

The implementation and the translation-expansion of research to model pricing and reimbursement policies would help to advance the field of genomic testing, setting an important precedent in a time of considerable ambiguity, uncertainty and heterogeneity in genomic medicine policies.

The main issue in this policy paper is to definitively show that patient outcomes and targeted therapies are decisive in improving patient care and are far superior to the current hit-or-miss attempts currently practiced. This, plus proven pathway-based approach, defined precisely by the patient's genotype information, will eventually be the most cost-effective approach to clinical practice, along with well-educated caretakers into this being the standard of care, hopefully then driving down costs. Then and only then will this approach become standardized by all, and not just at the major academic medical centers.

\section{Acknowledgements}

This work represents the outcome of a Genomic Medicine Alliance Public Health Genomics Working Group project over pricing and reimbursement of genomic tests, with a special focus on the EU countries.

\section{Disclosure Statement}

The authors declare no conflict of interests.

\section{References}

1 Personalized Medicine Coalition (PMC): The Case for Personalized Medicine, ed 4. Washington, Personalized Medicine Coalition, 2014.

-2 Crawford JM, Aspinall MG: The business value and cost-effectiveness of genomic medicine. Pers Med 2012;9:265-286.

$\checkmark 3$ Davis JC, Furstenthal L, Desai AA, Norris T, Sutaria S, Fleming E, Ma P: The microeconomics of personalized medicine: today's challenge and tomorrow's promise. Nat Rev Drug Discov 2009;8:279-286.
4 Mitropoulou C, Mai Y, van Schaik RH, Vozikis A, Patrinos GP: Stakeholder analysis in pharmacogenomics and genomic medicine in Greece. Public Health Genomics 2014;17: 280-286.

5 Mai Y, Koromila T, Sagia A, Cooper DN, Vlachopoulos G, Lagoumintzis G, Kollia P, Poulas K, Stathakopoulos V, Patrinos GP: A critical view of the general public's awareness and physicians' opinion of the trends and potential pitfalls of genomic testing in Greece. Pers Med 2011;8:551-561.
6 Mai Y, Mitropoulou C, Papadopoulou XE, Vozikis A, Cooper DN, van Schaik RH, Patrinos GP: Critical appraisal of the views of healthcare professionals with respect to pharmacogenomics and personalized medicine in Greece. Pers Med 2014;11:15-26.

7 Mette L, Mitropoulos K, Vozikis A, Patrinos G: Pharmacogenomics and public health: implementing 'populationalized' medicine. Pharmacogenomics 2012;13:803-813.

8 Prainsack B: Personhood and solidarity: what kind of personalized medicine do we want? Pers Med 2014;7:651-657. 
9 Williams MS: The public health genomics translation gap: what we don't have and why it matters. Public Health Genomics 2012;15: 132-138.

10 Williams MS: The genetic future: can genomics deliver on the promise of improved outcomes and reduced costs? Background and recommendations for health insurers. Dis Manag Health Outcomes 2003;11:277-290.

-11 Williams MS: Insurance coverage for pharmacogenomic testing. Pers Med 2007;4:479487.

12 The Office of Inspector General (OIG): Memorandum Report: Coverage and Payment for Genetic Laboratory Tests, Department of Health and Human Services (DHHS). OEI07-11-00011. Washington, The Office of Inspector General, 2012.

13 The Office of Inspector General (OIG): Payments for Laboratory Tests: Comparing Medicare, State Medicaid, and Federal Employees Health Benefits Programs, Department of Health and Human Services (DHHS). OEI-07-11-00010. Washington, The Office of Inspector General, 2013.

14 Merchant M: Pricing and Reimbursement Strategies for Diagnostics: Overcoming Reimbursement Issues and Navigating the Regulatory Environment. London, Business Insights, 2010.

15 Department of Health and Human Services (DHHS): Coverage and Reimbursement of Genetic Tests and Services: Report of the Secretary's Advisory Committee on Genetics, Health, and Society (SACGHS). Washington, Department of Health and Human Services, 2006.

16 National Institutes of Health: Reimbursement Models to Promote Evidence Generation and Innovation for Genomic Tests, Workshop Summary. Bethesda, National Institutes of Health, 2012.

17 Britnell M: In Search of the Perfect Health System. Basingstoke, Palgrave Macmillan, 2015.

-18 Miller I, Ashton-Chess J, Spolders H, Fert V, Ferrara J, Kroll W, Askaa J, Larcier P, Terry PF, Bruinvels A, Huriez A: Market access challenges in the EU for high medical value diagnostic tests. Pers Med 2011;8:137-14812.

19 Velasco Garrido M, Børlum Kristensen F, Palmhøj Nielsen C, Busse R: Health Technology Assessment and Health Policy-Making in Europe. Current Status, Challenges and Potential. Brussels, European Observatory on Health Systems and Policies, 2008.
20 Institute of Medicine: Conflict of Interest in Medical Research, Education, and Practice. Washington, National Academies Press, 2009.

21 Kwon S: Positive Listing of Pharmaceuticals. Health Policy Monitor, 2007. http://www. hpm.org/survey/kr/a9/2.

22 European Commission: European Workshop on Genetic Testing Offer in Europe, EU Scientific and Policy Report by the Joint Research Centre of the European Commission, Report EUR 25684 EN. Luxembourg, Publications Office of the European Union, 2013.

23 Patrinos GP, Baker DJ, Al-Mulla F, Vasiliou V, Cooper DN: Genetic tests obtainable through pharmacies: the good, the bad and the ugly. Hum Genomics 2013;7:17.

24 Pavlidis C, Lanara Z, Balasopoulou A, Nebel JC, Katsila T, Patrinos GP: Meta-analysis of nutrigenomic biomarkers denotes lack of association with dietary intake and nutrient-related pathologies. OMICS 2015;19:512-520.

25 Pavlidis C, Patrinos GP, Katsila T: Nutrigenomics: a controversy. Appl Transl Genomics 2015;4:50-53.

26 European Commission: Guidelines on a Medical Device Vigilance System, E.U. DG Health and Consumers (SANCO) Directorate B-Consumer Affairs Unit B2 - Health Technology and Cosmetics, MEDDEV 2.12-1 rev 8. Luxembourg, Publications Office of the European Union, 2013.

27 Evans JP, Watson MS: Genetic testing and FDA regulation: overregulation threatens the emergence of genomic medicine. JAMA 2015; 313:669-670.

28 Council of Europe: Recommendation CM/ $\operatorname{Rec}(2010) 11$ of the Committee of Ministers to member states on the impact of genetics on the organisation of health care services and training of health professionals, adopted by the Committee of Ministers on 29 September 2010 at the 1094th meeting of the Ministers' Deputies. Strasbourg, Council of Europe, Committee of Ministers, 2010.

29 Dierking A, Schmidtke J, Matthijs G, Cassiman J-J: The EuroGentest Clinical Utility Gene Cards continued. Eur J Hum Genet 2013;21:1.

30 European Commission: Recommendation on Cross Border Genetic Testing of Rare Diseases in the European Union. Brussels, EC Commission Expert Group on Rare Diseases, 2015.

31 Kierkegaard P: E-prescription across Europe. Health Technol 2013;3:205-219.

32 Personalized Medicine Coalition (PMC): The Adverse Impact of the US Reimbursement System on the Development and Adoption of Personalized Medicine Diagnostics, Issue Brief. Washington, Personalized Medicine Coalition, 2010.
33 Personalized Medicine Coalition (PMC): The Future of Coverage and Payment for Personalized Medicine Diagnostics. Washington, Personalized Medicine Coalition, 2014.

34 Berwouts S, Fanning K, Morris MA, Barton DE, Dequeker E: Quality assurance practices in Europe: a survey of molecular genetic testing laboratories. Eur J Hum Genet 2012;20: 1118-1126.

35 Berwouts S, Morris MA, Dequeker E: Approaches to quality management and accreditation in a genetic testing laboratory. Eur J Hum Genet 2010;18(suppl 1):S1-S19.

36 The European Personalized Medicine Association: Personalised Medicine in Europe Enhancing Patient Access to Pharmaceutical Drug-Diagnostic Companion Products, $\mathrm{Pa}$ tient Access Study. Berlin, Brüggenjürgen Institute for Social Medicine, Epidemiology, and Health Economics, Charité - Universitätsmedizin, 2014.

37 Kampourakis K, Vayena E, Mitropoulou C, Borg J, van Schaik RH, Cooper DN, Patrinos GP: Key challenges for next generation pharmacogenomics. EMBO Rep 2014;15:472476.

38 Reydon TA, Kampourakis K, Patrinos GP: Genetics, genomics and society: the responsibilities of scientists for science communication and education. Pers Med 2012;9:633643.

- 39 Snyder SR, Mitropoulou C, Patrinos GP, Williams MS: Economic evaluation of pharmacogenomics: a value-based approach to pragmatic decision making in the face of complexity. Public Health Genomics 2014;17:256-264.

40 Coulter A, Jenkinson C: European patients' views on the responsiveness of health systems and healthcare providers. Eur J Public Health 2005; 15:355-360.

41 Crews KR, Hicks JK, Pui C-H, Relling MV, Evans WE: Pharmacogenomics and individualized medicine: translating science into practice. Clin Pharmacol Ther 2012;92:467-475.

-42 Mai Y, Mitropoulou C, Papadopoulou XE Vozikis A, Cooper DN, van Schaik RH, Patrinos GP: Critical appraisal of the views of healthcare professionals with respect to pharmacogenomics and personalized medicine in Greece. Pers Med 2014;11:15-26.

43 National Academies of Sciences, Engineering, and Medicine: Applying an Implementation Science Approach to Genomic Medicine: Workshop Summary. Washington, National Academies Press, 2016.
Test Pricing and Reimbursement in Genomic Medicine
Public Health Genomics 2016;19:352-363 